\title{
Integrierte Versorgung - \\ Vom Hoffnungsträger zum \\ Ladenhüter der deutschen \\ Gesundheitspolitik?
}

VOLKER E. AMELUNG

UND SASCHA WOLF

Prof. Dr. Volker E. Amelung, Vorstandsvorsitzender, Bundesverband Managed Care e. V., Institut für Epidemiologie, Sozialmedizin und Gesundheitssystemforschung, Medizinische Hochschule Hannover

Dr. Sascha Wolf, Geschäftsführer, Bundesverband Managed Care e. V.

\author{
Das deutsche Gesundheitswesen befindet sich in \\ keiner Krise: Die medizinische Versorgung gehört \\ immer noch zu den besten der Welt. Nicht Revolution, \\ sondern Evolution lautet daher das Gebot der \\ Stunde. Der entscheidende Treiber für notwendige \\ Strukturveränderungen ist weder die Politik noch \\ sind es die Akteure des Gesundheitswesens, \\ sondern es sind die Versorgungsherausforderungen \\ selbst, die einen kontinuierlichen Prozess hin \\ zu integrierten und kooperativen Modellen \\ erzwingen. Aufgabe der Politik ist es, den Akteuren \\ ausreichende Handlungsmöglichkeiten zu geben, \\ Anreize für Investitionen zu setzen sowie die \\ Rahmenbedingungen schrittweise nachzubessern.
}

Es ist still geworden um die Integrierte Versorgung in Deutschland. Noch bis vor kurzem dominierte sie als Hoffnungsträger für mehr Qualität und Effizienz im Gesundheitswesen die politische Diskussion zur Weiterentwicklung der Gesetzlichen Krankenversicherung. Doch die Situation hat sich geändert: Am 01. Januar 2012 ist das Versorgungsstrukturgesetz in Kraft getreten - und die Integrierte Versorgung spielt praktisch keine Rolle. Dabei entsprechen die Ziele der Reform - Verbesserung der Verzahnung der Leistungssektoren, Flexibilisierung und Regionalisierung der vertragsärztlichen Vergütung, Stärkung wettbewerblicher Instrumente etc. - exakt den zentralen Elementen berufsfeld- und sektorenübergreifender Versorgungsmodelle. Wie ist es möglich, dass die Bundesregierung an den Zielen der Integrierten Versorgung festhält, sie als Instrument jedoch kaum weiter fördert und einsetzt?

Die Gründe mögen darin liegen, dass die Politik in gewisser Weise ratlos ist, wie der Integrierten Versorgung endlich zum Durchbruch verholfen

In gewisser Weise ist die Politik ratlos, wie der Integrierten Versorgung endlich zum Durchbruch verholfen werden kann.

werden kann. Die Gesetzgebung hat in der Vergangenheit schrittweise die Gestaltungsoptionen für die Akteure des Gesundheitssystems vermehrt. Mittlerweile besitzen Krankenkassen und Leistungserbringer durchaus weit- 


\begin{tabular}{|c|c|c|c|c|c|}
\hline Country & $\begin{array}{l}\text { Test results/records } \\
\text { not available at } \\
\text { appointment or du- } \\
\text { plicate tests ordered }\end{array}$ & $\begin{array}{l}\text { Key information } \\
\text { not shared among } \\
\text { providers }\end{array}$ & $\begin{array}{l}\text { Specials lacked } \\
\text { medical history or } \\
\text { regular doctor not } \\
\text { informed about } \\
\text { specialist care }\end{array}$ & $\begin{array}{l}\text { Experienced gaps } \\
\text { in Hospital/surgery } \\
\text { discharge planning }\end{array}$ & $\begin{array}{l}\text { Reported regular } \\
\text { doctor seemed } \\
\text { uninformed about } \\
\text { hospital/surgery } \\
\text { care }\end{array}$ \\
\hline AUS & 19 & 12 & 19 & 55 & 18 \\
\hline CAN & 25 & 14 & 18 & 50 & 19 \\
\hline FRA & 20 & 13 & 37 & 73 & 15 \\
\hline GER & 16 & 23 & 35 & 61 & 17 \\
\hline NETH & 18 & 15 & 17 & 66 & 9 \\
\hline NZ & 15 & 12 & 12 & 51 & 19 \\
\hline NOR & 22 & 19 & 25 & 71 & 18 \\
\hline SWE & 16 & 18 & 20 & 67 & 35 \\
\hline SWI & 11 & 10 & 9 & 48 & 15 \\
\hline UK & 13 & 7 & 6 & 26 & 11 \\
\hline US & 27 & 17 & 18 & 29 & 12 \\
\hline
\end{tabular}

Tabelle 1: Ausgewählte Ergebnisse in Prozent: Commonwealth Fund International Health Policy Survey of Sicker Adults in Eleven Countries 2011.

Quelle: Schoen et al 2011.

greifende Handlungsspielräume für selektivvertragliche, kooperative und sektorenübergreifende Versorgungsformen. Einen nennenswerten Anteil an der Gesamtversorgung haben diese bislang jedoch nicht erreichen können. Geht man auf die Suche nach den Blockaden, stößt man zumeist auf die vermeintliche Unbeweglichkeit der körperschaftlichen Strukturen und die Investitionsrisiken, die Konflikte zwischen niedergelassenen und stationär tätigen Ärzten sowie die Ängste vor dem Verlust der Selbstständigkeit und die Sorge um Transparenz von Qualitätsmängeln. Diese Ansätze bergen sicherlich einen Kern Wahrheit in sich und tragen dazu bei, die große Vorsicht zu erklären, mit denen die Akteure des Gesundheitswesens neuen Vertragsformen begegnen. Im Endeffekt handelt es sich hierbei jedoch nur um die Symptome der mangelhaften Rahmenbedingungen und der ungenügenden Anreize, sich an innovativen Versorgungsmodellen zu beteiligen oder in sie $\mathrm{zu}$ investieren.

\section{Warum brauchen wir mehr Integrierte Versorgung?}

Die Ausgaben in allen hochentwickelten Industrienationen haben eines ge- meinsam: Sie sind sehr ungleich verteilt. Der größte Teil der Aufwendungen wird stets durch eine nur vergleichsweise kleine Gruppe von Patienten verursacht. In den USA entfallen fast 90 Prozent der Gesamtausgaben auf die 30 Prozent der Bevölkerung mit dem schlechtesten Gesundheitszustand (Cohen, Yu 2010). In Deutschland ist die Situation ähnlich: Nach den Zahlen des GEK-Gesundheitsreports verursachen 20 Prozent der Versicherten 91,5 Prozent der Gesamtausgaben, 10 Prozent über 80 Prozent, 2,5 Prozent der Versicherten mehr als die Hälfte und die Hochkostenfälle $(0,5$ Prozent der Versicherten) über 20 Prozent (Gmünder Ersatzkasse 2003). Diese Ungleichverteilung könnte zur Folge haben, dass zukünftig die Gesundheitsausgaben spürbar ansteigen werden. Denn der medizinisch-technologische Fortschritt führt dazu, dass immer mehr Indikationen behandelbar, aber nicht unbedingt heilbar sind. Verknüpft mit der demographischen Alterung der Bevölkerung bedeutet dies, dass die Anzahl von Menschen mit komplexen und chronischen Krankheitsbildern aller Voraussicht nach zunehmen wird. Sind heute
46 Prozent aller Erkrankungen chronischer Art, werden es im Jahr 2020 rund 60 Prozent sein (Gensichen et al 2006). Gleichzeitig steigt die Multimorbidität. Die hinter chronischen Erkrankungen liegende Wertschöpfungskette bzw. die unterschiedlichen Wertschöpfungsketten bei Multimorbiden sind äußerst komplex und binden nicht nur unterschiedliche Haus-, Fach- und Krankenhausärzte, sondern gegebenenfalls auch Leistungserbringer der Rehabilitation oder der Pflege ein.

\section{Zur zunehmenden Komplexität trägt auch das rasant wachsende medizinische Wissen bei.}

Zur zunehmenden Komplexität trägt auch das rasant wachsende medizinische Wissen bei. Enthielt die Muster-Weiterbildungsordnung 1976 der Bundesärztekammer noch 27 Gebietsbezeichnungen, 14 Teilgebietsbezeichnungen und 15 Zusatzbezeichnungen, so ist deren Anzahl mittlerweile auf 32 Gebiete mit über 30 Facharzt- und Schwerpunktkompetenzen sowie knapp 50 Zusatz- 
Weiterbildungen angestiegen (Bundesärztekammer 2010). Beides zusammen - zunehmende Multimorbidität und Komplexität der Erkrankungen sowie fortschreitende Subspezialisierung der Leistungserbringer - erfordern funktionierende Informationsflüsse zwischen den Akteuren und sinnvoll aufeinander abgestimmte Prozessketten.

Das typisch deutsche Phänomen einer weitgehend gelebten Sektorentrennung ist mit der Bewältigung dieser Herausforderung zu großen Teilen überfordert (Schlette et al 2005). In einer aktuellen internationalen Vergleichsstudie von elf Ländern (Tabelle 1) geben 23 Prozent der befragten Patienten in Deutschland an $(\mathrm{N}=1.200)$, dass der Informationsfluss zwischen den Leistungserbringern ungenügend ist. Das sind mehr als in jedem anderen betrachteten Staat. Doch nicht nur hier befindet sich die Gesetzliche Krankenversicherung in der Negativ-Spitzengruppe: so beklagen beispielsweise 35 Prozent, dass Fachärzte die Krankenhistorie des Patienten ungenügend kennen bzw. die Grundversorger über die Behandlungen der Fachärzte nicht informiert sind (Schoen et al 2011). Insgesamt führen das Fehlen einer prozess- und informationstechnischen Verzahnung zweier voneinander getrennter Versorgungssektoren und die damit einhergehende Parallelität von personellen und apparativen Kapazitäten zu vermeidbaren Effektivitäts- und Effizienzverlusten (Ulrich 2002).

\section{Der Anteil berufsfeld- und sektorenübergreifender Versorgungsmodelle stagniert}

Die Ineffizienz des deutschen Gesundheitswesens ist wahrlich kein neues Thema. Der mangelhafte Informationsaustausch zwischen den Leistungserbringern, der ursächlich ist für das Problem der Doppel- und Mehrfachuntersuchungen sowie für Brüche in der Kontinuität der Behandlung beim Wechsel zwischen ambulantem und stationärem Sektor, ist seit Jahrzehnten bekannt. Deshalb war es richtig, dass die Gesundheitspolitik in den vergangenen knapp 15 Jahren einem kontinuierlichen Entwicklungspfad gefolgt ist: Mehr Wettbewerb zwischen den Krankenkassen, und zwar nicht allein über den Beitragssatz, sondern auch über Versorgungskonzepte, mehr Wahlmöglichkeiten für die Versicherten und Patienten und mehr unternehmerischen Spielraum für die Akteure in den unterschiedlichen Sektoren des Gesundheitswesens (Amelung 2008). Seit dem 2. GKV-Neuordnungsgesetz 1997 hat der Gesetzgeber über eine ganze Reihe von Reformen einen Flickenteppich an vertraglichen Möglichkeiten geschaffen. Dabei lassen sich zwei Gruppen unter den besonderen Versorgungsformen unterscheiden: Einerseits die Strukturverträge nach $\mathbb{}$ 73a SGB V, die hausarztzentrierte Versorgung nach $\mathbb{\int} 73 \mathrm{~b}$ SGB V sowie die besondere ambulante ärztliche Versorgung nach $\mathbb{}$ 73c SGB V, die zwar Kooperationen zwischen Kostenträgern und Leistungserbringern, jedoch keine sektorenübergreifende Koordination ermöglichen. Andererseits gestatten genau dies die Modellvorhaben nach $\mathbb{S} \$$ 63-65 SGB V, die Disease-Management-Programme nach $\mathbb{}$ 137f-g SGB V und eben die Integrierten Versorgungsformen nach $\mathbb{S} 140 \mathrm{a}-\mathrm{d}$ SGB V.

Mit der Neuformulierung des $\mathbb{1 4 0 a -}$ d SGB V durch das Gesundheitsmodernisierungsgesetz 2004 wurde erstmals eine sektorenübergreifende oder eine interdisziplinär-fachübergreifende Versorgung der Versicherten ohne expliziten Einbezug der Kassenärztlichen Vereinigungen ermöglicht. Stattdessen können nunmehr einzelne Ärzte und Ärztenetze im Rahmen des Einzelvertragssystems direkte Vertragspartner der Krankenkassen werden. Zudem ist es den Krankenkassen erlaubt, auch mit Managementgesellschaften, die nicht direkter Versorger sind, zu kontrahieren. Die konkrete Ausgestaltung der Verträge zu Inhalt und Vergütung bleibt weitestgehend den Vertragspartnern überlassen.

Eine weitere Aufwertung erfuhr die Integrierte Versorgung mit dem GKVWettbewerbsstärkungsgesetz von 2007 und der Einbindung der Pflegeversicherung sowie mit dem Arzneimittelmarktneuordnungsgesetz von 2011, durch das auch pharmazeutische Unternehmen in den Kreis potenzieller Vertragspartner aufgenommen worden sind. Entgegen der Meinung vieler Kritiker, die daran zweifeln, dass die Berücksichtigung der Pharma-Industrie an der Integrierten Versorgung eine reelle Option darstellt, ist der erste bundesweite Vertrag bereits in Kraft: Knappschaft, Desitin Arzneimittel $\mathrm{GmbH}$ und die Managementgesellschaft Deutsche Gesundheitssystemberatung haben einen Vertrag zur medizinischen Versorgung von Kindern und Jugendlichen, die an Epilepsie leiden, abgeschlossen.

\section{Trotz der politischen Anstrengungen und erfreulicher Beispiele kommt die Integrierte Versorgung nicht wirklich in}

Doch trotz der politischen Anstrengungen und einiger erfreulicher Beispiele kommt die Integrierte Versorgung in Deutschland nicht wirklich in Fahrt. Nach dem Auslaufen der Anschubfinanzierung Ende 2008 sank die Zahl der Verträge von knapp 6.200 auf heute rund 5.000. Auch ihre ökonomische Bedeutung verharrt auf sehr geringem Niveau: 70 Prozent sind kleinere Kooperationen, die stark von einzelnen Beteiligten abhängen. Großflächig angelegte Verträge, die nicht nur die Integrierte Versorgung, sondern auch die Regelversorgung maßgeblich beeinflussen, sind eher Einzelfälle. Der Anteil der Krankenkassenausgaben für besondere Versorgungsformen an den Gesamtausgaben liegt durchschnittlich bei unter einem Prozent (Gersch 2011). Es ist ruhig geworden um die Integrierte Versorgung, ähnlich der Zeit nach ihrer erstmaligen Einführung im Jahr 2000.

\section{Versorgungsstrukturgesetz - kein Durchbruch, aber besser als sein Ruf}

Den Durchbruch für berufsfeld- und sektorenübergreifende Versorgungsmodelle wird auch das Versorgungsstrukturgesetz nicht bringen. Die jüngste Gesundheitsreform ist dennoch besser als ihr Ruf und schafft zumindest punktuell die Voraussetzungen für eine bessere Verzahnung der Leistungssektoren. So wird mit der Neuformulierung des \ 116b SGB V ein weiterer Sektor der »Ambulanten Spezialfachärztlichen Versorgung ", in welchem sowohl stationäre als auch vertragsärztliche Leistungsanbieter weitgehend ohne Zugangsrestriktionen gleichberechtigt und zu identischen Konditionen tätig werden können, 
etabliert (Deutscher Bundestag 2011). Dieser Ansatz ist durchaus zu begrüßen, denn bei allen berechtigten Befürchtungen hinsichtlich einer drohenden Mengenausweitung und unangemessen steigender Kosten aufgrund fehlender Steuerungsinstrumente, können hierdurch Reibungsverluste an den Schnittstellen zwischen den Versorgungsbereichen verringert werden. Insbesondere die Ergänzung des $\mathbb{S} 140 \mathrm{~b}$ SGB V, durch welche die Leistungserbringer nach \116b SGB V im Rahmen eines Vertrages zur Integrierten Versorgung zur Erbringung von Leistungen der Ambulanten Spezialfachärztlichen Versorgung berechtigt werden, eröffnet die Chance, dass gleichsam »durch die Hintertür» die sektorenübergreifende Versorgung angeschoben wird.

Auch die Verpflichtung der Krankenhäuser zu einem verbindlichen Entlassmanagement (Ergänzung $\mathbb{S} 39$ SGB V) fördert das Ineinandergreifen der unterschiedlichen Behandlungsstufen. Gerade in der stationären Nachversorgung hat sich in der Praxis gezeigt, dass bei Patienten mit chronisch entgleisten Grund- und Nebenerkrankungen das Zusammenspiel der Akteure im stationären und ambulanten Sektor noch nicht reibungslos funktioniert. Sollte es gelingen, mehr Teamorientierung, Ansprechpartner mit klar definierten Zuständigkeiten, einen funktionieren-

\section{Neue Versorgungsformen werden sich nur durchsetzen, wenn die Initiative zu großen Teilen von den Ärzten ausgeht.}

\section{Arztnetze - Wegbereiter der Integrierten Versorgung?}

Die Änderung des $\ 87 \mathrm{~b}$ SGB V ist auch deshalb von zentraler Bedeutung, als das hierdurch die Ärzte zur stärkeren Beteiligung an kooperativen Modellen motiviert werden könnten. Denn eines ist klar: Neue Versorgungsformen werden sich nur durchsetzen, wenn die Initiative zu großen Teilen von den Ärzten ausgeht. Aktuell ist bei den Leistungserbringern der Handlungsdruck zum Abschluss

den Informationsfluss sowie einheitliche Qualitäts- und Beurteilungskriterien im Entlassmanagement zu etablieren, dann würde das in der Tat die nachstationäre Behandlung verbessern und helfen, Therapiebrüche zu vermeiden. Solange die Frage der Anreize bzw. der Finanzierung jedoch nicht geklärt ist, bleibt abzuwarten, ob sich hier belastbare Strukturen wirklich durchsetzen werden.

Ein weiterer wichtiger Aspekt, der in der Öffentlichkeit kaum wahrgenommen worden ist, stellt die durch einen Antrag der Regierungskoalition nachträglich eingefügte Änderung des $\$ 87 \mathrm{~b}$ SGB V dar. Durch diesen erhalten die von Selektivverträgen ausgesprochen gering (Amelung 2011b). Auch wenn viele unzufrieden mit der Arbeitsbelastung und den Verdienstmöglichkeiten in der vertragsärztlichen Versorgung sind, so muss doch kaum ein Akteur befürchten, aus dem System herausgedrängt zu werden. Andererseits dominiert bei vielen die Angst, dass eine Veränderung der Versorgungsstruktur zu Kompetenzbeschneidung, Überwachung und Eingriff in die Therapiefreiheit führt. Umso erfreulicher ist es, dass anscheinend immer mehr Ärzte ihre Scheu vor kooperativen Verbünden ablegen (Amelung, Wolf 2011).
Zwar ist die Struktur des Sektors mit rund 140.000 Ärzten, von denen knapp 50.000 in Gemeinschaftspraxen tätig sind (Kassenärztliche Bundesvereinigung 2010), weiterhin atomistisch geprägt. Kooperationen zwischen den Niedergelassenen setzen sich jedoch immer mehr durch: Die Anzahl der Ärztenetze hat sich in den letzten 10 Jahren von 200 auf 400 verdoppelt, die der eingeschriebenen Ärzte von 10.000 auf 30.000 sogar verdreifacht (Agentur deutscher Ärztenetze 2011). Die Zahl der Netze und deren Professionalisierung werden aller Voraussicht nach weiter ansteigen. Im nächsten Schritt könnten überregionale oder nationale Ärzteketten folgen. Hinzu kommen regionale Vollversorgungsmodelle unter Einbezug der Krankenkassen wie beispielsweise die populationsorientierten Gesundheitsnetze solimed, QuE Nürnberg, Gesundes Kinzigtal, der PatientPartner Verbund in München auf Initiative der GMZ GmbH oder die Gesundheitsnetze prosper/proGesund.

\section{Mehr Mut in der Gesundheitspolitik - Vorbild Schweiz?}

Andere Länder sind bereits bedeutend weiter. So sind beispielsweise in der Schweiz die Hälfte aller Allgemeinmediziner, Internisten und Pädiater in Ärztenetzen eingeschrieben. 84 Prozent dieser Netze haben in ihren Verträgen mit den Krankenversicherern eine Budgetmitverantwortung vereinbart. Fast die Hälfte verfügt über vertraglich fixierte Kooperationen mit anderen Leistungserbringern, insbesondere den Krankenhäusern. Allein durch die Anwendung des Gatekeeping-Modells konnten laut neuerer Studien die Kosten pro Kopf um bis zu 19 Prozent gesenkt werden (Berchtold, Huber 2011).

Vielleicht gab das den Anstoß dafür, dass das Schweizer Parlament am 30. September 2011 mit nahezu Zweidrittel-Mehrheit eine Gesundheitsreform beschlossen hat, die aus deutscher Perspektive wahrlich revolutionäre Züge trägt: Der Anteil der Versicherten in Integrierten Versorgungsmodellen soll von heute 13 Prozent der Bevölkerung (rund eine Million Versicherte) auf 60 Prozent erhöht werden. Der Trick, mit dem diese Steigerung erreicht werden soll, ist so einfach wie brillant: Versicherte, die an 
der Integrierten Versorgung teilnehmen, sollen maximal 500 CHF als Selbstbehalt bezahlen, Versicherte in der traditionellen Versorgung hingegen bis $\mathrm{zu}$ 1.000 CHF (Nationalrat 2011). Verbunden mit der weiterhin geringeren Prämie für die Versicherten in Modellen der Integrierten Versorgung wird ein massiver ökonomischer Anreiz zur Beteiligung gesetzt. Kurzum: die "gesteuerte" Versorgung wird zur Regelversorgung, während die »ungesteuerte« Versorgung zum teureren Wahltarif wird.

Es bleibt nicht aus, dass bei einer solch umwälzenden Reform starke Widerstände auftreten. So haben sich in der Schweiz mehrere Initiativen gebildet, die das Vorhaben in einem Referendum

\section{In der Schweiz zeigt sich deutlich, wie wichtig der Erfolg der Netzstrukturen als Grundlage für die Integrierte Versorgung gewesen ist.}

kippen möchten. Zuversichtlich sollte jedoch stimmen, dass die Ärztenetze und der Berufsverband »Hausärzte Schweiz« die Vorlage des Parlaments unterstützen. Hier zeigt sich deutlich, wie wichtig der vorhergehende Erfolg der Netzstrukturen als Grundlage für die Integrierte Versorgung gewesen ist.

\section{Wie kann der Integrierten Versorgung in Deutschland zum Durchbruch verholfen werden?}

In Deutschland sind wir von dem Mut der Schweizer Reformen weit entfernt. Zwar herrscht über die effizienzfördernde Wirkung der Integrierten Versorgung nicht nur in der Politik, sondern auch unter den Akteuren des Gesundheitssystems ein weitreichender Konsens. Doch die überfällige Überwindung der Blockaden ist weiterhin nicht in Sicht. So richtig neue Instrumente wie die Ambulante Spezialfachärztliche Versorgung oder ein verpflichtendes Entlassmanagement auch sein mögen - eine grundsätzliche Überwindung der Sektorengrenzen wird nur gelingen, wenn die immer weitergehende Verkomplizierung und Zersplitterung des Gesamtsystems beendet wird und der Gesetzgeber seinen Fokus gezielt auf die Integrierte Versorgung ausrichtet. Noch immer fehlen schlüssi- ge Antworten auf die schwierigen Fragen der Sicherstellung, der Bedarfsplanung und einer praktikablen Budgetbereinigung. Insbesondere getrennte Budgets im ambulanten und stationären Bereich erzeugen den Anreiz für die Akteure des einen Sektors, zu Lasten der Akteure des anderen Sektors finanziell zu profitieren und lassen die Ziele einer Verbesserung der Behandlungsprozesse und einer sinnvollen sektorenübergreifende Ressourcenallokation in den Hintergrund treten (Glaeske 2002). Zudem müssen endlich die Voraussetzungen für eine elektronische Patientenakte geschaffen werden, die das Herzstück jedes gesteuerten Behandlungsablaufs und damit ein unverzichtbarer Bestandteil integrierter Versorgungsformen darstellt. An dem großflächigen Einsatz strukturierter Informations- und Kommunikationsinstrumente führt kein Weg vorbei. Hauptansatzpunkt sind jedoch die Akteure des Gesundheitssystems selbst: Wer der Integrierten Versorgung wirklich zum Durchbruch verhelfen will, der muss Krankenkassen, Leistungserbringer und Patienten wirkungsvoll zur freiwilligen Teilnahme motivieren.

\subsection{Krankenkassen}

Insbesondere die Krankenkassen tun sich schwer damit, ihre Rolle als möglicher Motor der Integrierten Versorgung anzunehmen. Die Erfahrungen in der Vergangenheit haben gezeigt, dass Zusatzbeiträge für sie existenzielle Auswirkungen haben können. Die hohen Anfangsinvestitionen für Projekte der Integrierten Versorgung bei mittel- bis langfristigem Return on Investment lassen sich nur schwerlich mit einem auf das Geschäftsjahr orientierten »Budgetdenken « und der Systemlogik von Körperschaften des öffentlichen Rechts vereinbaren. Die Planungen der Kassen werden zusätzlich durch den Gesundheitsfonds und dessen verzögerten Korrekturabrechnungen erschwert. Ein weiteres Problem ist die nicht praxisgerechte Budgetbereinigung zwischen Regelversorgung und Integrierter Versorgung. Theoretische und evaluierte Einsparungen entsprechen nicht der Realität dessen, was bei den Kassen ankommt. Insgesamt gesehen führt sowohl der Verhandlungs- und Investitionsaufwand als auch die Wechselwirkungen zur Regelversorgung dazu, dass der für die Integrierte Versorgung erforderliche Einsatz oft als unangemessen hoch eingestuft wird. Stattdessen ist es für die Krankenkassen häufig attraktiver, in große und flächendeckende Verträge nach $\mathbb{S} \mathbb{S} 73 \mathrm{~b}$ oder $73 \mathrm{c}$ SGB V zu investieren.

Umso wichtiger ist es, die Finanzhoheit der Krankenkassen wieder zu stärken und ihnen Investitionsplanungen für einen Zeitraum von drei bis fünf Jahren zu ermöglichen. Flankierend sollten Innovationsbudgets bei den Kassen eingeführt werden, die sich beispielsweise aus zwei Prozent der $\mathrm{Zu}-$ weisungen aus dem Gesundheitsfonds speisen. Die eine Hälfte des Budgets sollte für die Entwicklung hochinnovativer Versorgungsformen und Pilotprojekte eingesetzt werden, die andere Hälfte dient der Anschubfinanzierung von Verträgen zur Integrierten Versorgung. Sollte eine Krankenkasse in einem Jahr ihr Innovationsbudget nicht ausschöpfen, fließen die Mittel zurück in den Gesundheitsfonds. Der sachgerechte Einsatz der Mittel ist durch eine standardisierte Evaluation der geförderten Projekte oder eine Zertifizierung sicherzustellen, deren Hauptaugenmerk auf

\section{Ein weiteres Problem ist die nicht praxisgerechte Budgetbereinigung zwischen Regelversorgung und Integrierter Versorgung.}

der Hebung von Sparpotenzialen sowie der Steigerung von Versorgungsqualität und Patientenzufriedenheit liegen sollte. Schließlich muss die Krankenkasse ihre Gewinnpotenziale ausschöpfen und kalkulieren können. Hierzu bedarf es einer ausreichenden Budgetbereinigung ohne Einigungsvorbehalt sämtlicher Vertragspartner.

\subsection{Leistungserbringer}

Wie bereits dargestellt sind die Anreize für die Leistungserbringer zur Teilnahme an der Integrierten Versorgung zu gering. Einerseits besteht angesichts der 
heutigen Honorierungen in der GKV bei den meisten Ärzten kein wirtschaftlicher Handlungsdruck, sich an Selektivverträgen beteiligen zu müssen. Andererseits verbleiben ihnen aufgrund der bereits vorhandenen hohen Arbeitsbelastung kaum Kapazitäten für zusätzliche Aufgaben. Zudem fühlen sich viele Ärzte von der Komplexität der Verträge zur Integrierten Versorgung überfordert. Sie wird momentan von den Leistungserbringern dann favorisiert, wenn sie neue Wege in der medizinischen Versorgung ermöglicht. Nur eine qualitätsorientierte Vergütung motiviert den Arzt zur bestmöglichen Versorgung des Patienten und forciert den angestrebten Qualitätswettbewerb. Die Veränderung des \87b SGB V ist daher der richtige Weg. Durch teilweise Übertragung des Morbiditätsrisikos auf den Arzt und einer Gewinnbeteiligung werden Mengenausweitungen ein wirkungsvoller Riegel vorgeschoben.

\subsection{Versicherte}

Auch die Versicherten stehen neuen Versorgungsformen eher skeptisch gegenüber (Amelung 2009). Die meisten fühlen sich in der Regelversorgung gut

\section{An mehr standardisierten Evaluationen und Veröffentlichung der Ergebnisse führt kein Weg vorbei.}

aufgehoben und vertrauen der vermeintlich vom Staat gewährleisteten Qualität. Da die Vorteile innovativer Versorgungsformen erst bei Inanspruchnahme im konkreten Krankheitsfall erkennbar werden, besteht bei ihnen kaum Bereitschaft für alternative Versorgungsangebote. Die Versicherten sollten daher durch mehr Transparenz zu einer Beteiligung motiviert werden. Aktuell können sie aufgrund mangelnder Informationen die unterschiedlichen Angebote der Krankenkassen kaum beurteilen. An mehr standardisierten Evaluationen und Veröffentlichung der Ergebnisse führt daher kein Weg vorbei. Mit steigender Transparenz wird auch die Bedeutung neuer Versorgungsformen als Wettbewerbsinstrument für die Krankenkassen zunehmen und ein dynamischer
Entwicklungsprozess in Gang gesetzt.

\section{Schlussfolgerungen}

Wenn chronische Erkrankungen und Multimorbidität die zentralen zukünftigen Herausforderungen des Gesundheitssystems darstellen, dann müssen traditionelle Strukturen der Gesetzlichen Krankenversicherung teilweise aufgebrochen werden. Denn das fragmentierte deutsche Gesundheitssystem ist kaum in der Lage, die hohen Anforderungen, die komplexe Krankheitsbilder an einen ganzheitlichen Behandlungsablauf und patientenorientierte Koordinierung der medizinischen Versorgung stellen, effizient zu erfüllen. Umso bedauerlicher ist es, dass trotz umfassender Bemühungen des Gesetzgebers in den vergangenen 15 Jahren der Anteil sektoren- und berufsfeldübergreifender Versorgungskonzepte stagniert. Das ernüchternde Beharrungsvermögen der Akteure hat vielfältige Ursachen und reicht von schwer kalkulierbaren Investitionsrisiken bei den Krankenkassen über Kooperationsvorbehalte der Leistungserbringer bis hin zum Zögern vieler Versicherten, die allseits bekannte und durchaus gute Regelversorgung gegen neue, aus individueller Sicht qualitativ schwer einschätzbare Versorgungsformen einzutauschen.

Vor diesem Hintergrund wird auch das Versorgungsstrukturgesetz keinen Durchbruch für die Integrierte Versorgung bringen. Zwar werden mit Einführung der Ambulanten Spezialfachärztlichen Versorgung, einem verbindlichen Entlassmanagement für Krankenhäuser und gesonderten Vergütungsregeln für Arztnetze punktuell die Voraussetzungen für eine bessere Verzahnung der Leistungssektoren geschaffen, die grundlegenden Schnittstellenprobleme jedoch nicht behoben. Hierfür wäre es notwendig, die Rahmenbedingungen in den jeweiligen Sektoren aneinander anzupassen. Die schwierigen Fragen der Sicherstellung, der Bedarfsplanung und einer funktionierenden Budgetbereinigung dürfen nicht länger aufgeschoben werden.
Die Erfolge von Managed Care in der Schweiz und die zunehmende Ausbreitung von Arztnetzen in Deutschland zeigen, dass Kooperation und Koordination medizinischer Leistungen möglich sind und sich durchsetzen, wenn die beteiligten Vertragspartner die Chance auf einen persönlichen Mehrwert sehen.

\section{Literaturangaben}

Agentur deutscher Ärztenetze (2011): URL http:// www.deutsche-aerztenetze.de, Download: 04.10.2011.

Amelung, V.E. (2008): Managed Care Modell der Zukunft? In: Amelung, V.E., Meyer-Lutterloh, K., Schmid, E., Seiler, R., Lägel, R., Weatherly, J.N. (Hrsg.): Integrierte Versorgung und Medizinische Versorgungszentren. Berlin: MWV, 2. Auflage, S. 1-34.

Amelung, V.E. (2009): Managed Care Neue Wege im Gesundheitsmanagement. In: Amelung, V.E., Deimel, D., Reuter, W., van Rooij, N., Weatherly, J.N. (Hrsg.): Managed Care in Europa. Berlin: MWV, S. 3-30.

Amelung, V.E. (2011a): Managed Care Neue Wege im Gesundheitsmanagement. Wiesbaden: Gabler, 5. Auflage.

Amelung, V.E. (2011b): Neue Versorgungsformen auf dem Prüfstand. In: Amelung, V.E., Eble, S., Hildebrandt, H. (Hrsg.): Inno vatives Versorgungsmanagement. Berlin: MWV, S. 3-16

Amelung, V.E., Wolf, S. (2011): Gesundheitssystem im Umbruch - Ärztenetze: Treiber für integrierte Versorgung? In: Der Urologe; 50, S. 1566-1572.

Berchtold, P., Huber, F. (2011): Schweizer Ärztenetze - ein Beitrag zur Integrierten Versorgung. In: Amelung, V.E., Eble, S., Hildebrandt, H. (Hrsg): Innovatives Versorgungsmanagement. Berlin: MWV, S. 91-99.

Bundesärztekammer (2010): (Muster-) Weiterbildungsordnung 2003 in der Fassung vom 25.05.2010. URL: http://www.bundesaerztekammer.de/page.asp?his=1.128.129, Download: 12.12.2011.

Cohen, S.B., Yu, W. (2010): The concentration and persistence in the level of health expenditures over time: estimates for the U.S. population, 2007-2008. In: Agency for Healthcare Research and Quality; Statistical Brief No. 309. 
Deutscher Bundestag (2011): Beschlussempfehlung und Bericht des Ausschusses für Gesundheit zu dem Gesetzentwurf der Bundesregierung »Entwurf eines Gesetzes zur Verbesserung der Versorgungsstrukturen in der gesetzlichen Krankenversicherung ". Berlin: Drucksachen 17/8005

Gensichen, J., Muth, C., Butzlaff, M., Rosemann, T., Raste, H., Müller de Cornejo, G. et al. (2006): Die Zukunft ist chronisch - Das Chronic Care Modell in der deutschen Primärversorgung. In: Zeitschrift für ärztliche Fortbildung und Qualität im Gesundheitswesen; 10, S. 365-374.

Gersch, M. (2011): Ouo Vadis IV? - Monitoring Integrierte Versorgung. Deutsche Gesellschaft für Integrierte Versorgung, mimeo Berlin.

Glaeske, G. (2002): Integrierte Versorgung in Deutschland - Rahmenbedingungen für mehr Effektivität und Effizienz? In: Preuß, K.J., Räbiger, J., Sommer, J. (Hrsg.): Managed Care: Evaluation und Performance-Measurement integrierter Versorgungsmodelle. Stuttgart New York: Schattauer Verlag, S. 3-19.

Gmünder Ersatzkasse (2003): GEK-

Gesundheitsreport 2003-Schwerpunkt:

Charakterisierung von Hochnutzern im Gesundheitssystem. In: Schriftenreihe zur Gesundheitsanalyse, Band XXVI.

Hensgen, R. (2000): Integrierte Versorgung - Innovation oder Rückbesinnung auf historische Wurzeln. In: Das Krankenhaus; 4. S. $275-280$.

Kassenärztliche Bundesvereinigung (2009): Grunddaten 2009/2010 zur vertragsärztlichen Versorgung in Deutschland. URL: http://www.kbv.de/publikationen/125.html, Download: 12.12.2011.

Nationalrat (2011): Bundesgesetz über die Krankenversicherung. Teilrevision. Managed Care. URL: http://www.parlament.ch/ab/frameset/d/n/4820/367211/ d n $4820 \quad 367211 \quad 367374$.htm, Download: 12.12.2011.

Schlette, S., Knieps, F., Amelung, V.E. (2005): Versorgungsmanagement für chronisch Kranke. Bonn: Kompart.

Schoen, C., Osborn, R., Squires, D., Doty, M., Pierson, R., Applebaum, S. (2011): New 2011 Survey Of Patients With Complex Care Needs In Eleven Countries Finds That Care Is Often Poorly Coordinated. In: Health Affairs, No. 12.

Ulrich, S. (2002): Ordnungspolitische, vertragliche und wettbewerbsrechtliche Aspekte neuer autonomer Versorgungsstrukturen im deutschen Gesundheitswesen. Göttingen: Cuvillier Verlag.

\section{Effektive \\ Gesundheitsversorgung}

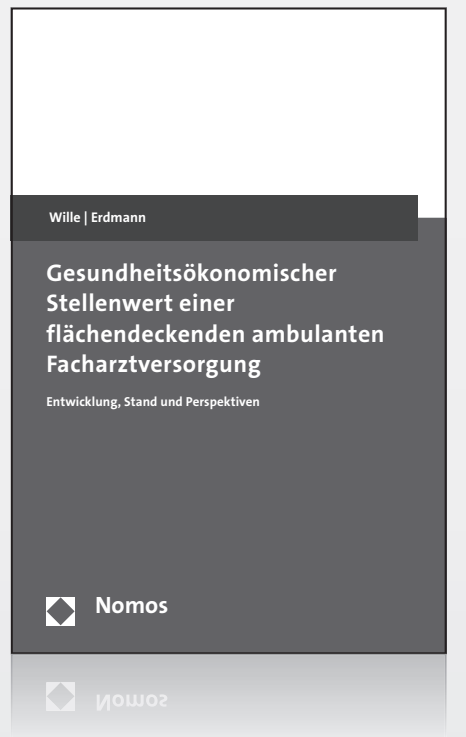

Den Kern der Arbeit bildet eine Vorausberechnung der Nachfrage nach niedergelassenen Fachärzten in Baden-Württemberg und in Bayern vor dem Hintergrund der demographischen Entwicklung. Den Abschluss bildet eine ordnungspolitische Analyse, welche die Voraussetzungen einer effizienten und effektiven Gesundheitsversorgung an der Sektoren-Schnittstelle herausstellt.

Weitere Informationen: www.nomos-shop.de/13837

\section{Gesundheitsökonomischer Stellenwert einer flächendeckenden ambulanten Facharztversorgung}

Entwicklung, Stand und Perspektiven

Von Eberhard Wille und Daniel Erdmann

2011, 231 S., brosch., 49,- $€$

ISBN 978-3-8329-6795-6
Nomos 